\title{
Remote Control: The Natural Language of Architecture
}

FRANK JACOBUS

University of Arkansas

"The fundamental problem of communication is that of reproducing at one point either exactly or approximately a message selected at another point." -Claude Shannon ${ }^{1}$

\section{INTRODUCTION}

The architectural design process is a means of translating information into form, and has long relied on indirect ("remote") control mechanisms for communicating and translating the architect's authorial intent into a built work. These methods have generally evolved from a more direct, physical basis, as both technology and the discipline have evolved. To communicate design ideas, architects have relied on methodologies that range from an extreme desire for control, to models that attempt to relinquish many controls entirely. Early communication models, in part due to lack of material, form, and program diversity, allowed for a less systematic and complex descriptive method; inscriptions in the earth, physical detail models along with a set of instructions, or simple scale models of the intention were all that was required. ${ }^{2}$ As cultures and their technologies advanced, communication methods such as scaled orthographic drawings, specifications and other forms of written instructions, and now fully realized Building Information Models, have become normative practice in a profession that looks for total control of the built work before it is physically realized. Apart from the communicative control models mentioned above, there are authorial models which have also progressed in complexity and abstraction alongside societal advancements. In the discipline's infancy, authorship involved subtle evolutions of proportion and order within a well-established typological system. In modernism, the authorial models evolved as architects experimented with increased typological invention in response to a radically changing technological and social environment. Advancing to the contemporary "digital" moment, architects continue to develop systems to control complexities within the work, mapping strategies that deal with collecting and spatializing data, while others see contemporary design tools as a means to relinquish some design control to outside forces whose unexpected potential is compelling. This paper gives examples of remote communicative and authorial controls, and posits a new theory of the potential meaningful effects of leveraging these control mechanisms in new ways using three projects by SILO AR+D.

\author{
MARC MANACK \\ University of North Carolina at Charlotte
}

\section{INFORMATION AND INCREASING ABSTRACTION}

In his book The Information, James Gleick describes the evolution of attempts at recording information from more physical models to those with a radically increased sense of abstraction. In speaking of language evolution he states, "marks, images, pictographs, petroglyphs - as these forms grew stylized, conventional, and thus increasingly abstract, they approached what we understand as writing, but one more transition was crucial, from the representation of things to the representation of spoken language: that is, representation twice removed. There is a progression from pictographic, writing the picture; to ideographic, writing the idea; and then logographic, writing the word. ${ }^{13}$ There is a form of "Remote Control" in abstraction itself, resulting from human evolution which experiences increasing levels of abstract engagement (or disengagement) with the physical world. As communication evolves, it does so from a more evident physical basis to a more abstract basis. ${ }^{4}$ These abstractions signify a distance, remoteness from the physical thing itself, to phonetic translation of the thing using an alphabetic language. This is what Gleick describes as "abstracting information away from its physical substrate." ${ }^{5}$ Gleick describes writing as coming into being to "retain information across time and across space. Before writing, communication is evanescent and local; sounds carry a few yards and fade to oblivion." 6 The same evolution of communicative models exists in architecture in the form of the drawing and the specification. Drawings are abstract descriptions that are meant to retain information across time and space; an effort at communicating a position and intention about something that must be mediated through an abstract form, different from the desired thing itself. The information models that Gleick describes, and the mechanisms used to translate them from one form to another, share an affinity with those in the architectural realm. Architects have accepted a distinct level of divorce from the physical nature of the object in order to possess the power of abstract communicative tools.

\section{COMMUNICATIVE REMOTENESS}

Architecture was conceived as fundamentally mediated through the drawing and the specification. ${ }^{7}$ Alberti made clear in his treatise on architecture that an architect's drawings were "the original act of creation," and that the "physical building that may follow is only a copy." ${ }^{\prime \prime}$ In the authorial and communicative models discussed above, remote control has always played an essential yet evolving role in the process of designing and describing a work (predominantly through 
drawing) that is intended to be physicalized. The spirit of remoteness exists in Alberti's words. The word "remote" implies a happening from a distance, and the allowance, whether by choice or by exigency, of external forces to play a role in design. The extent of remoteness becomes an indication of how pronounced a role these external forces are allowed to play. Robin Evans has asserted that this lack of control is the "peculiar disadvantage under which architects labor; never working directly with the object of their thought, always working at it through some intervening medium, almost always the drawing." ${ }^{\prime \prime}$ The foundation of architecture's discipline is about a how architects articulate and structure this "intervening medium," to communicate relationships between information and building, to make design conception become built form. Attaching the word "Remote" to the word "Control" implies a distancing, and starts to suggest a more passive form of authorship. As long as the drawing is a mediator, the architect is never in complete control, and we can read one trajectory within the field as an attempt to assert control of the object. For reasons that range from creative integrity to risk management, architects typically attempt to assert as much control as possible, choosing to minimize instability in the design process so to gain as much control over the object to be built as possible. In turn, as our technology has evolved, at least in normative practice, architects have persistently developed tools, whether drawings or digital models, which control the final built work to the greatest extent possible.

While architects have attempted to gain control of their remoteness in the translation of information to building, they have also developed parallel methods of communicating design intent. Alongside the drawing, the diagram has evolved as a preferred method of communicating this intent; whether to the designer themselves or to a larger audience. Diagrams are fundamentally reductive and abstract machines that act as translation mechanisms of language into built form. In order to be affective, diagrams must remove large amounts of information so to increase flexibility, focus, and agency with respect to the information that remains. ${ }^{10}$ Diagrams are reductions that help organize relations..$^{11}$ In this way, and certainly in relation to Gleick's description of language evolution above, much of human thought and language is "diagrammatic." The act of defining is in many ways an act of diagramming; and to expect clear definition and clear distinction is to have a sensibility toward the diagram. Diagrams are not a singular entity with a fixed purpose. It would be impossible to describe the diagram as a phenomenon that exists in only one state, apart from its situational context. Being that they are reductive, diagrams are abstractions of ideas; meaning they are the result of an exclusion of some information in the service of the clarity of other privileged information. In this way it is possible to think or act "diagrammatically" without offering any physical artifact for one's visual consumption. A diagram's abstraction is what empowers it, and is also what establishes its remoteness.
Deleuze suggests that we are beyond a simple defining of diagrams as a mode of representation and need to consider them as maps; a "cartography that is coextensive with the whole social field."12 Giovanni Corbellini in Diagrams, instructions for Use cites synthesis as "one of the primary functions of the diagram"; describing it as a tool that compresses the process of design. ${ }^{13}$ For Corbellini, it is the diagram's ability to compress, both as a generative and representational tool, which makes it such an appropriate device in a design environment radically affected by an increased complexity of programmatic criteria. Because the diagram has a mathematical basis and reductive tendencies, it embodies a mathematical logic and becomes machinic in its nature. Gilles Deleuze refers to the diagram appropriately as an "abstract machine" ${ }^{14}$, and Corbellini follows by referring to diagrams as "true machines for thinking." 15 Sanford Kwinter shares Deleuze and Corbellini's view when he describes objects as a "composition of forces" arriving via a "compositional event" that is a formulation of the diagram as a dynamic "engine of novelty." ${ }^{16}$ For Kwinter, the diagram has the capacity to both uncover and generate novel qualities of objects, or object "events" themselves, by decoding the underlying object forces and reusing them as a generative mechanism. The diagrams abstract operative mode becomes a powerful form of remoteness that allows it to lose sight of the physical, personal nature of the object itself, in favor of a compression and acceleration of ideas.

Fundamentally, what distinguishes architectural design from other creative practice is that an architect's projects are shaped by real forces (codes, POR's, budgets, zoning) that remain largely beyond our control, which we must absorb, challenge, and ultimately sublimate in the work. The inflections of form are largely pre-determined before the architect even gets the project, and easily outweigh the influence of the architect's ambitions for compositional arrangement in the project's development. In fact, embracing these external forces as elements that act as remote controls within an architectural process will elicit new responsive formal characteristics.

\section{AUTHORIAL REMOTENESS}

In his book, Design Methods, Kari Jormakka describes various forms of authorial controls that come from outside the discipline of architecture. For instance, music, language, and natural form are all external ideas brought to architecture through various periods to institute an external (remote) control mechanism. Jormakka describes how the gothic builders use quadrature as an armature to be built into - this is simultaneously a form of control and acceptance of a lack thereof. In other words, these builders didn't use scale drawings (because there was no agreed upon system of measurement at the time) but quadrature gave the builders an assurance of proportion and order - that which fell between became less controlled which was acceptable due the overlying order on which the system was based. ${ }^{17}$ In this system an overall proportional control 
was demanded but other forms of control were relinquished to the sculptors and masons. In other examples of authorial remoteness Jormakka discusses the Surrealist techniques for subverting authorship. In each example the Surrealist artists employ methods for relinquishing control that allow for results that couldn't have been conceived of without this subversive process. ${ }^{18}$ For the Surrealists, these were methods to subvert consciousness in order to discover new forms that would not have emerged otherwise.

Numerous other forms of authorial remoteness have existed through the history of architecture as well, especially throughout the twentieth-century. Christopher Alexander's book, A Pattern Language, accepts a form of design remoteness coming from pre-established, vetted spatial typologies that could be repurposed for similar new needs. ${ }^{19}$ Peter Eisenman's formal diagrams, Bernard Tshumi's event diagrams, and Rem Koolhaas' program diagrams all possess their own forms of remoteness which allow processes, often only loosely managed by the architect, to determine final form. These, and other varieties of typological thinking, are examples of remoteness that assume a lexicon of historically established typologies that can be mined and often hybridized to constitute new form. ${ }^{20}$

Similar examples exist outside of architecture and speak to a larger cultural desire to question the role of authorship in art and the design disciplines. In a show titled "40 years of Rule-Based Art," work was exhibited that used "one or more logic-based systems to direct the design and creation of the object." ${ }^{21}$ In each of the pieces shown in the exhibit the artist created a script that could then be performed by someone else. In "Abstract Painting, Blue" for instance, Ad Reinhardt creates a script by which the painting should be made. An excerpt from the created script reads as follows:

"...one formal device, one color-monochrome, one linear division in each direction, one symmetry, one texture, one free-hand brushing...no lines or imaginings, now shapes or composings or representings, no visions or sensations or impulses...nothing that is not of the essence." 22

In this rule-based approach Ad Reinhardt is relinquishing some of the minutia of control over to a craftsperson, maker, or simply an "other." 23 Musicians such as John Cage, Pierre Boulez, and Earle Brown are well known for experiments of indeterminacy in their work. Some of Earle Brown's scores for instance act as maps to be decoded by performers as they play. Brown uses a loose, rule-based structure in this work, such that the decoding of these maps is left up to the performer's interpretation, which creates variance and uniqueness within each new performance. Much of John Cage and Pierre Boulez's work used related diagrammatic strategies to stand in the place of the musical score (which itself was already a form of diagram). Cage used matrices, charts, and squares as devices to order sound, embracing indeterminacy, flexibility, and outside interpretation for the work's performers. The divorce of self-conscious control became a mechanism for Cage that subverted pre-established taste and fixity in the made object. ${ }^{24}$ Through a variety of techniques that use indeterminacy, musicians like Cage and Boulez became liberated from the minutia of decision making regarding every note which allowed their focus to be on a larger conceptual intelligibility. ${ }^{25}$

These examples scratch the surface of the multitude of ways that architects have been indebted to techniques of authorial remoteness as architectural generators, but teach us that learning to embrace ideas of indeterminacy helps us find connections between things we might not have imagined otherwise. Indeterminacy enables us to break down our preconceptions and allows us to accept risk; embracing accidental discoveries within generative systems. This is an intellectual investment in an understanding of the processes that translate information into form. By embracing "Remote Control" architects may become less interested in a designing of things and more interested in a designing of modes. "Remote Control" is a form of diagrammatic practice that harnesses the capacity to deal with contingency in the production of new form. It also becomes the type of practice that allows room for the experimental translation of the world of information into the world of form.

\section{SILO AR+D - BEYOND CONTROL}

Over the past three years the firm SILO AR+D has designed and built three pavilions which embrace and take pleasure in the notions of remote control discussed above.

Barn Again was inspired by the photography of Falling Barns in the Ozark region, reclaimed wood from a barn that was slated to be torn down was used for a spatial installation at the Fay Jones School of Architecture + Design. The design of the installation reconciles two consistently oppositional qualities in the barn photos, the persistence and resilience of the iconic barn figure, and the filigreed volume of wood members suspended in animation during the process of decay. The overall form was a lofted extrusion of a gambrel end barn form to the fallen form that existed in the photographs; an initial use of "Remote Control" as formal driver. This form was then triangulated and the pieces were sized in relation to each other in a parametric modeling environment. A script was developed that would not only rationalize the geometry into a limited number of segment lengths, but would color code each segment by length, and ultimately produce a set of shop drawings for construction. Instead of using the CNC Router to cut the individual pieces the students manually cut them using traditional woodworking equipment. The students that built the installation had not designed it, and so did not know what the final product was to ultimately look like. This released the constructors from attempting to create an idealized image, and allowed them to progress expeditiously. 


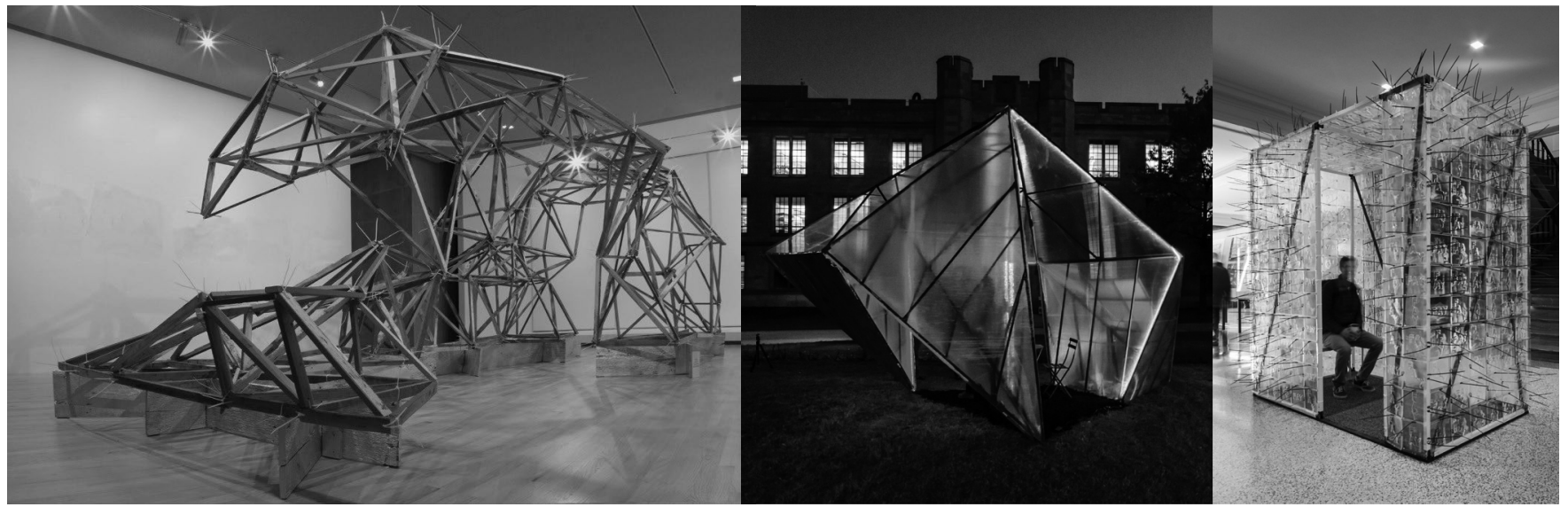

Figure 1 Barn Again (Left), Super Sukkah (Middle), Fayz Box (Right) all by SILO AR+D Saskpower Building, interior view of entrance lobby showing original mosaic finishes and illuminated ceiling. Undated, Saskpower Archives

To insure the complex shape could be built quickly, students and faculty collaborated to develop a computational process that would allow the installation to be fabricated and assembled by hand. One piece at a time, line segment by line segment, the shape emerged as the color coded pieces were accordingly zip tied together. The zip-tie as a structural joint, and the loose nature of the board ends, allowed flexibility in construction that affected the overall form. Essentially a selfsupporting, largely tensile structure, the final form was found only after the last piece was installed. The relinquishing of control in this project came in several forms. First, the overall form was determined by the lofting of two preexisting form and so was an accepted indeterminate. Next, the individual joints allowed the students to adjust and rework the form where need be to establish the structural rigidity necessary. Lastly, the color coding of pieces was employed as a tool for rapid assembly but accepted as a resulting aesthetic, beyond the control of the designers.

The Super Sukkah was built as part of an open national competition. This project involved the design and construction of a temporary pavilion that attempted to rethink the architecture of a traditional Jewish Sukkah. The specific competition theme asked designers to generate spaces that exist between absence and presence. The design transforms the Sukkah into a three-dimensional shelter with a distinct day and night presence. Through the perceptual affects that result from surface reflection, the day-time phenomenon creates an environmental absence as the Super Sukkah becomes a chameleon that reflects its surroundings. The night presence of the Super Sukkah is a geometric inversion that provides a new figural character defined by the illumination of the interior. The triangulated geometry creates a structurally interconnected volume whose surfaces continuously changes scale, orientation, and alignment as one moves around it. Through this process the triangles became pyramidal shapes, each one resting against the next, creating two distinct exterior and interior figures. Given the schedule, constraints, and modest budget, our strategy was to deploy a minimal amount of material to create a maximum amount of space, with a simplified construction method. The student team took a leadership role in determining the logistics for construction and implementation and chose to prefabricate modules of the tube steel structure in Fayetteville, Arkansas that would be bolted together and skinned in the field. Additionally, the students engineered the dynamic LED lighting system which was powered by a photovoltaic cell, and programmed to illuminate at sunset, and stay lit until the solar power captured and stored during the day, dissipated. In this pavilion we experimented with design remoteness by allowing a predetermined geometry (in this case the Star of David) to be a geometric point of departure that would be unfolded to create the necessary volume. We also treat light as material in this structure and accept a constantly changing random pattern of light that creates an evolving nighttime presence that was primarily out of our control.

Fayz Box is a booth for recording video testimonials built for the Fay Jones School of Architecture + Design. The dean required that the Fayz Box be able to be deconstructed, stored easily, and reconstructed quickly throughout the academic year. To accomplish this, the project was developed as a knock-down kit of components that could be assembled by a layman with limited knowledge of the project. The logistical constraints meant that the construct would be a light frame and a thin envelope, and the design became an experiment with the superimposition of competing patterns of formal and material content that create depth in the flat; an attempt to project substantial volume within a skin. Each layer has its own logic of fabrication, organization, and articulation, which not only produce an array of translucent material effects, but take advantage of the portfolio of tools available at the school. The acrylic tiles, are silkscreened with a variety images from the school's historical archive, and embossed with dynamic versions of the school's "FAY" logo. The veil-like effect of the coffered pattern is a nod to the "ballet modernism" of Edward Durrell Stone, a Fayetteville native. The final tile configuration is not designed, but determined indirectly 
by the students during construction by selecting tiles from randomly collated piles, allowing for the creation of a unique installation each time it is built, and subsequently re-built.

Each of the projects mentioned above explored the use of instructions rather than drawings in the construction process; an exploration of how to relinquish of some design controls and produce a myriad of remotely authored spatial effects. The act of making contributed to the design of an undetermined final form. In these cases "Remote Control" suggests a way of dealing with the contingencies of a transdisciplinary world, integrating the act of creation with the act of collaboration. Instead of asserting increasing amounts of control, the most powerful possibilites in contemporary architectural practice are methods that animate the architect's distance from the final object, instigating unique collective forms of authorship. These projects show that the authorial relationships don't have to be high-tech, but instead emerge from deeper understanding and embracing of the architect's natural relationship to objects they shape; redeploying the communicative tools of architecture to do so.

\section{CONCLUSION}

Technology continues to advance abstract communication methods and resituate how architects establish the translation of idea to form. In theorizing control systems in architecture, we must make a distinction between the remoteness of communicative mechanisms and the remoteness of authorial control, even though these two phenomena seem inextricably linked. As communicative abstraction advance in the discipline of architecture so too does the desire and speculation for new forms of authorial control. Projects that involve relaxed authorial controls take pleasures in the unknowns of the resultant form. Increased abstraction will involve further speculation on the nature of the origin of form. Architects will continue to reverse engineer architecture to uncover the elemental nature of information into effect. This parsing of the informational origin of form will allow transdisciplinary influence to more profoundly affect the spatial constitution of architecture such that all information, even that well outside the discipline's bounds, will be subject to conversion into architectural form. The nature of interdisciplinary work in contemporary critical practice is such that we must learn to handle greater levels of contingency and loss of control in the design process; a willingness to relinquish our overt desire for authority, and embrace architecture's natural language of remote control.

\section{ENDNOTES}

1. Gleick, James. The Information. New York, NY: Vintage, 2011, 3.

2. Carpo, Mario. The Alphabet and the Algorithm. Cambridge, MA: MIT Press, 2011.

3. Gleick, James. The Information. New York, NY: Vintage, 2011, 32.

4. Gleick, James. The Information. New York, NY: Vintage, 2011, 13.

5. Gleick, James. The Information. New York, NY: Vintage, 2011, 109

6. Gleick, James. The Information. New York, NY: Vintage, 2011, 31.

7. Vidler, Anthony. "Diagrams of Utopia." Daidalos, no. 74 (200): 6. 8. Carpo, Mario. "The Art of Drawing." Architectural Design: Drawing
Architecture, no. 225 (September/ October 2013): 128-30.

9. Evans, Robin. The Projective Cast: Architecture and its Three Geometries. Cambridge, MA: MIT Press, 1995.

10. Phillips, Alan. "The Free Library." thefreelibrary.com. Accessed August 19, 2011. Last modified January 1, 2006. http://www.thefreelibrary.com/

11. Corbellini, Giovanni. "Diagrams: Instructions for Use." Lotus International, no. 127 (January 2006): 93.

12. Deleuze, Gilles. "Foucault." University of Minnesota Press (1986/88) 34.

13. Corbellini, Giovanni. "Diagrams: Instructions for Use." Lotus International, no. 127 (January 2006): 92.

14. Deleuze, Gilles. "Foucault." University of Minnesota Press (1986/88) 34.

15. Corbellini, Giovanni. "Diagrams: Instructions for Use." Lotus International, no. 127 (January 2006): 92.

16. Kwinter, Sanford. "The Hammer and the Song." Diagram, no. 48 (January 1998): 33.

17. Jormakka, Kari. Basic Design Methods. Boston, MA: Birkhauser, 2008, 14-15.

18. Jormakka, Kari. Basic Design Methods. Boston, MA: Birkhauser, 2008, 37.

19. Jormakka, Kari. Basic Design Methods. Boston, MA: Birkhauser, 2008, 45.

20. Jormakka, Kari. Basic Design Methods. Boston, MA: Birkhauser, 2008, 53.

21. Glimcher, Marc and Rose, Bernice, "40 Years of Rule-Based Art."

22. Glimcher, Marc and Rose, Bernice, "40 Years of Rule-Based Art."

23. Galanter, P., Generative Art and Rules-Based Art, Vagueterrain.net. June, 2006.

24. Potter, Keith, "Earle Brown in Context," The Musical Times, Vol. 127, No. 1726. December, 1986. Pp. 681-683.

25. Bochner, Mel, "The Serial Attitude," Artforum, 6:4, December 1967, p.25. 